Article

\title{
Nano-Scale Characterization of a Piezoelectric Polymer (Polyvinylidene Difluoride, PVDF)
}

\section{Hyungoo Lee ${ }^{1}$, Rodrigo Cooper ${ }^{1}$, Ke Wang ${ }^{2}$ and Hong Liang ${ }^{1, *}$}

1 Department of Mechanical Engineering, Texas A\&M University, College Station, Texas 778433123, USA; E-Mails: prettylhg@tamu.edu; rcooper@tamu.edu

2 Department of Physics, Texas A\&M University, College Station, Texas 77843-3123, USA; E-Mail:kephwk@tamu.edu;

* Author to whom correspondence should be addressed; E-Mail: hliang@tamu.edu; Tel.: +1-979-862-2623; Fax: +1-979-845-3081

Received: 9 October 2008; in revised form: 6 November 2008 / Accepted: 12 November 2008 / Published: 18 November 2008

\begin{abstract}
The polymer polyvinylidene difluoride (PVDF) has unique piezoelectric properties favorable for Micro-Electro-Mechanical Systems (MEMS) and Nano-ElectroMechanical Systems (NEMS) applications. In the present research, we conducted nanometer-length scale characterization of this material using several high-resolution techniques. Specifically, we used an atomic force microscope (AFM) to study the nanoand microstructures of the PVDF under stress and to measure their nanoscale conductivity and piezoelectricity. We found that the surface morphology, electronic structure, and microstructure are profoundly affected under electrical potential. Such a behavior is important for the properties and performance of MEMS and NEMS.
\end{abstract}

Keywords: Polyvinylidene difluoride (PVDF), piezoelectricity, ferroelectricity, conductivity, atomic force microscope (AFM).

\section{Introduction}

Piezoelectric materials play an important role for Micro-electro-mechanical systems (MEMS) and Nano-electro-mechanical systems (NEMS) [1, 2]. Polyvinylidene difluoride (PVDF) has been widely used in engineering applications due to its favorable chemical and mechanical properties [3-7]. 
Properties such as high piezoelectric coefficient, good flexibility, biocompatibility [8-10], low acoustic and mechanical impedance, and light weight, are especially unique for MEMS applications. The polymer PVDF is one of the most widely used piezoelectric materials in the fluoropolymer family. Its piezoelectricity was discovered in 1969 [11]. However, the mechanism of piezoelectricity has not been clearly explained [12-14]. This uncertainty has hindered the development of MEMS or NEMS.

Many researchers have attempted to explain the origin of the piezoelectricity of the polymer [15-19] and to investigate the structure change of PVDF due to electric field [19] and temperature [20]. Recently, development in new characterization techniques, particularly nanoscale analysis, has made it possible to bring new insights from the piezoelectricity measurements. One of these techniques is Atomic Force Microscopy, which enables characterization of surface morphology at the nano-scale. Using this technique, we have investigated the relationship between the piezoelectric properties and structures of PVDF. An electrical field was applied to the PVDF samples. The effects of the external electrical voltage on the sample surface were observed using an atomic force microscope (AFM). The responding time of the PVDF samples to mechanical stress was studied.

\section{Experiments}

\subsection{Materials}

This study was performed using three different samples of uniaxially oriented polyvinylidene difluoride (PVDF) films. The original PVDF film samples were uniaxially stretched to obtain the polar $\beta$-phase. The samples were examined for polarity ( $\beta$-phase, TTT type) and piezoelectric coefficient $(23 \mathrm{pC} / \mathrm{N})$. Using a metal evaporator, the metal coatings were deposited on the films as electrodes. Two samples with thickness of $110 \mu \mathrm{m}$ and one with $52 \mu \mathrm{m}$ were coated with $28 \mu \mathrm{m}$ of $\mathrm{Ag}, 600 \AA$ of $\mathrm{NiCu}$, and $150 \mathrm{~nm}$ of $\mathrm{Au}$, respectively. The size of all samples was $3 \mathrm{~cm}$ in length and $1 \mathrm{~cm}$ width.

\subsection{AFM and the System Setup}

A home-built splitter, i.e., a Shark box, was attached to an AFM. The splitter distributes electrical potentials and passes a current from samples to a picoameter. The splitter was powered through the AFM's built-in power supply as shown in Figure 1. The sample surface is scanned with a standard $\mathrm{Si}_{3} \mathrm{~N}_{4}$ probe. The surface profile is measured against the electrical potential with respect to the principal direction $\left(\mathrm{d}_{31}\right)$ of the PVDF films.

When bent, a PVDF sample generates a micro-ampere current flowing to a picoameter. A computer is connected to the picoameter to record the output current using the LabView software. In this case, conductive AFM probes were used. 
Figure 1. Diagram for the experimental setup and characterization. This work was done with a Si3N4 probes. The external electrical potentials were supplied to the PVDF samples throughout the shark box which is a splitter for electrical potentials and current.

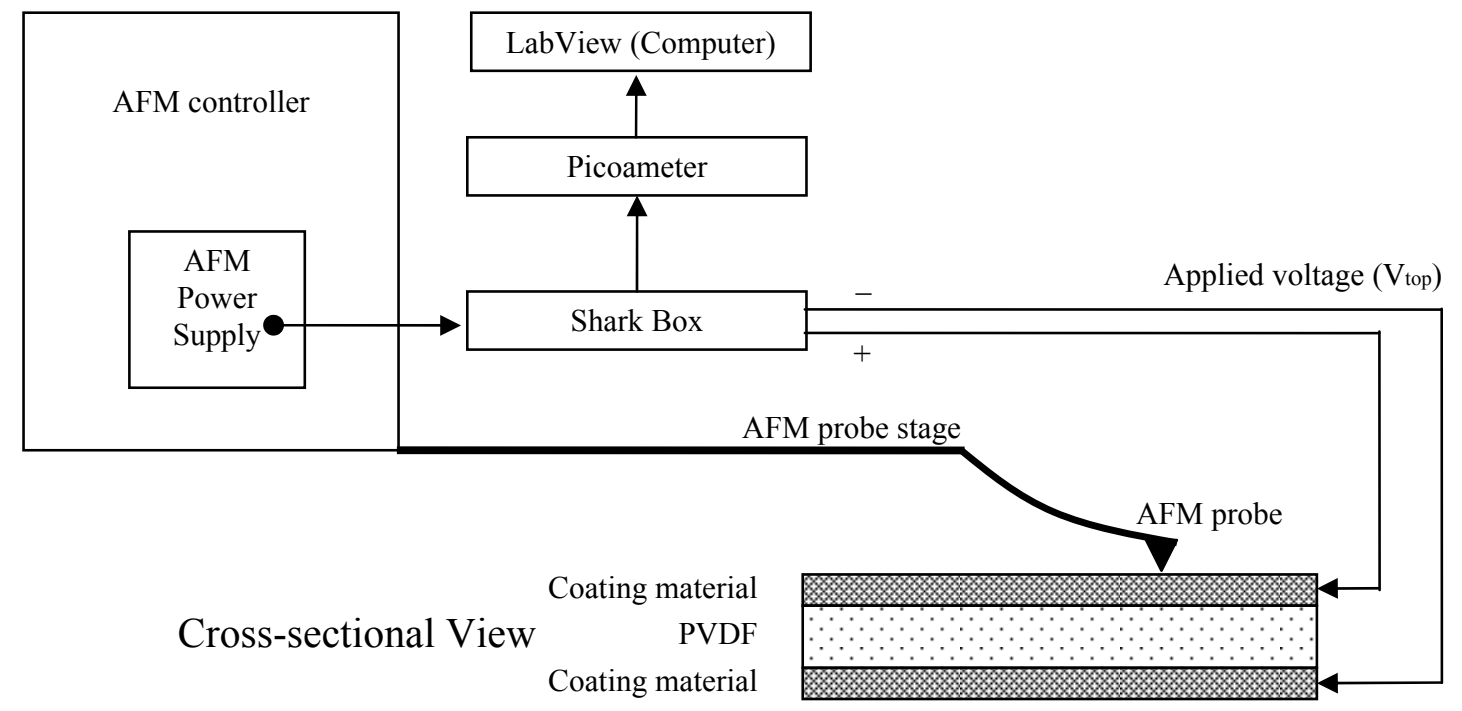

\subsection{Motorized Linear Stage (MLS)}

A motorized linear stage (MLS) was used to characterize the responding time of the dipoles in PVDF. The coated PVDF samples were placed on the MLS from one end to the other as shown in Figure 2(a) and (b). With one of the sample holders fixed in a stationary position, the other holder moved reciprocally at a frequency of $4 \mathrm{~Hz}$ in a stroke length of $3 \mathrm{~cm}$.

Figure 2. Simple diagrams of Motorized Linear Stage (MLS) (a and b) and AFM setup (c) to apply mechanical stresses on a sample.

(a)

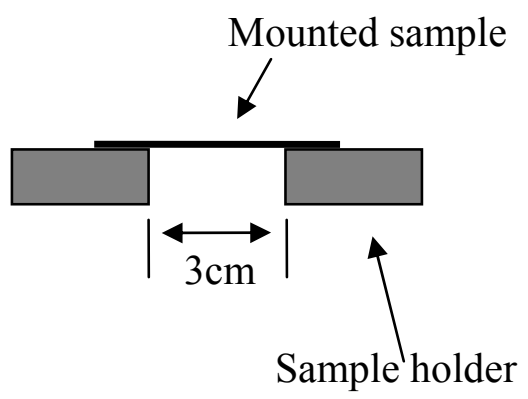

(b)

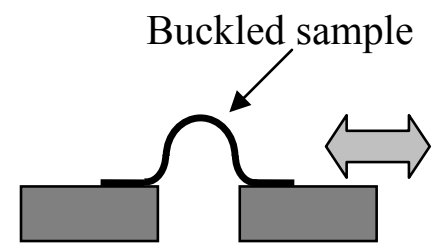

(c)

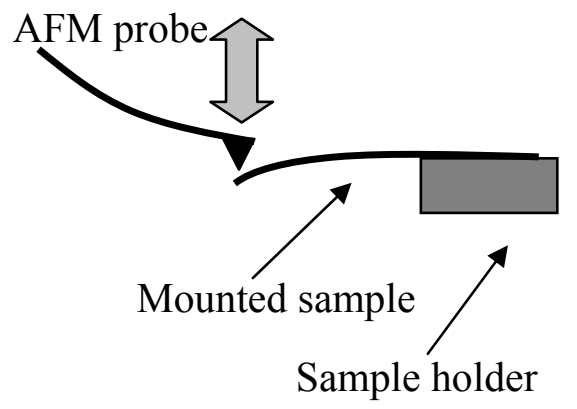

The reciprocal motion induced buckling over PVDF samples. Figure 2(c) shows the AFM setup applying a force on one end. The PVDF sample produced an electrical potential due to the 
piezoelectric property. The voltage generated was recorded using the LabView through a picoameter. MSL and AFM probe bending tests were conducted with respect to the principal direction $\left(\mathrm{d}_{31}\right)$ of the PVDF films.

\section{Results}

Scans of a PVDF sample, with the thickness of $110 \mu \mathrm{m}$ and an Ag-coating of $28 \mu \mathrm{m}$, were conducted using the AFM with a standard $\mathrm{Si}_{3} \mathrm{~N}_{4}$ probe. Figure 3 (a) shows a scanned image of the sample prior to connecting the Shark box.

Figure 3. AFM images for the PVDF sample surface which was $110 \mu \mathrm{m}$ thick with $28 \mu \mathrm{m} \mathrm{Ag}$ coated without (a) or with (b) connection of electric field. (c) The profiles of the two scans along the dashed line

(a) $V_{\text {top }}=$ no connection

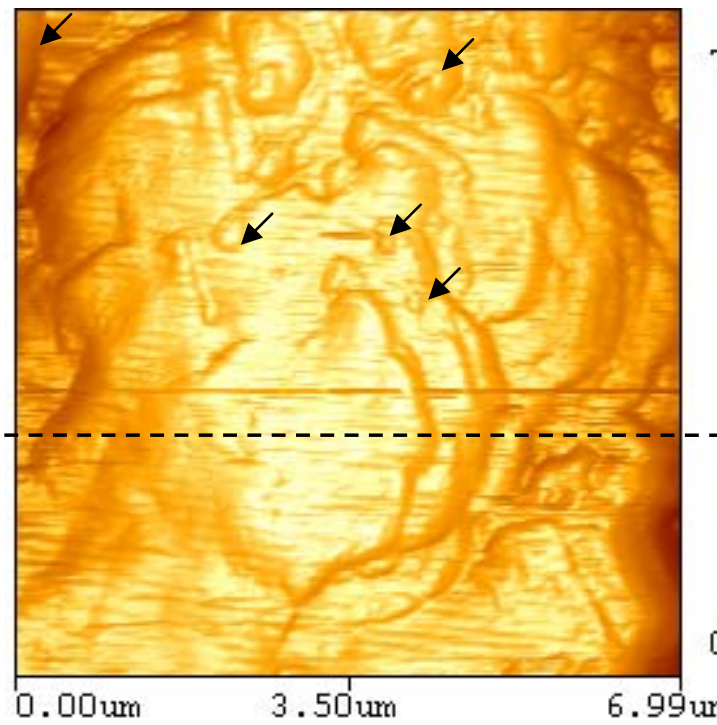

(b) $\mathrm{V}_{\text {top }}=0 \mathrm{~V}$

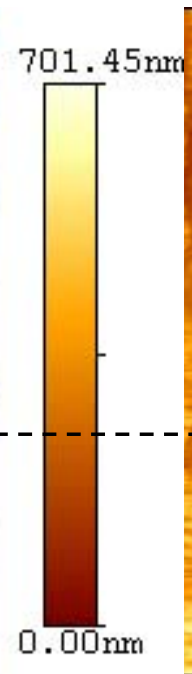

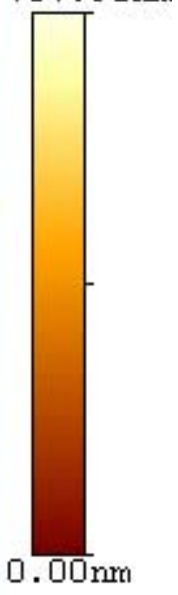

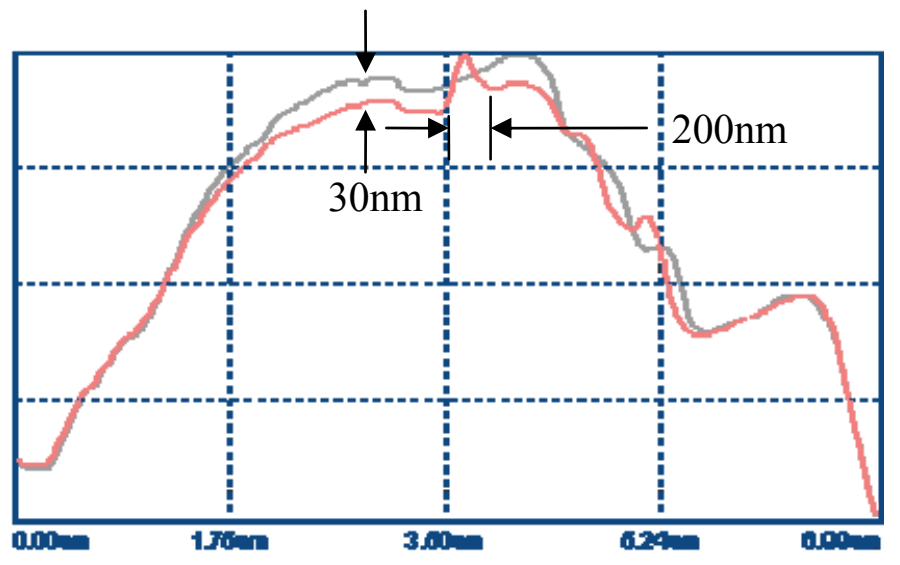

(c) Profile

$-\mathrm{V}_{\text {top }}=$ no connection

$-\mathrm{V}_{\text {top }}=0 \mathrm{~V}$ 
The same region of the sample was scanned with the leads of the shark box connected to the PVDF as shown in Figure 1 while an applied electrical potential was at $0 \mathrm{~V}$. The profiles of the two scans along the dashed line are shown in Figure 3 (c). It is seen that at the zero potential, the surface profile underwent a morphology change resulting in an increase in roughness.

Figure 4. Roughness change with the applied voltage.

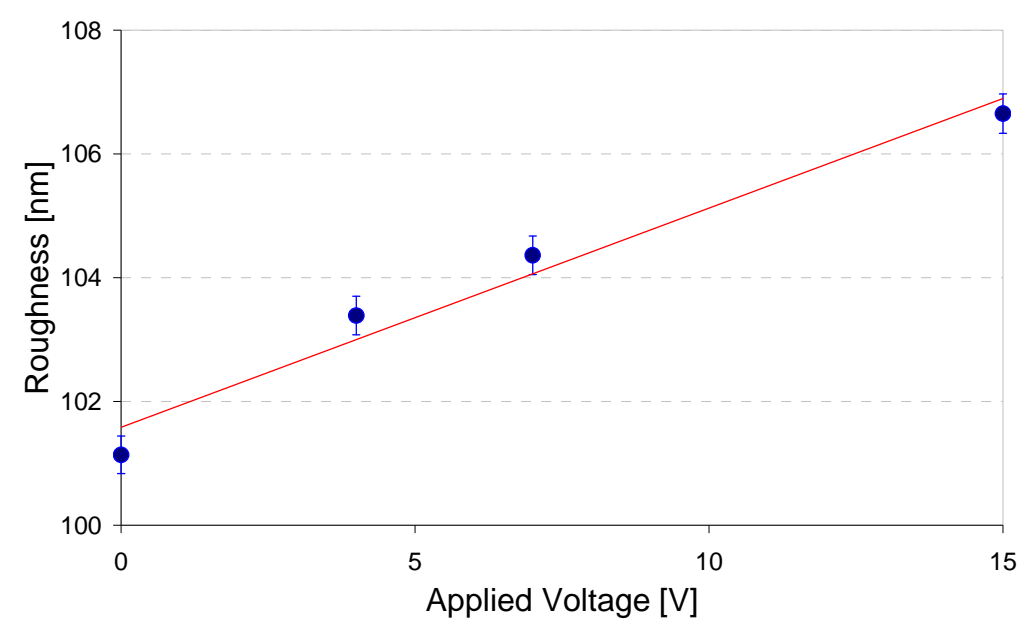

As the electric potential on the PVDF is increased in discrete steps of $0,4,7$, and $15 \mathrm{~V}$, the roughness $\left(\mathrm{R}_{\mathrm{a}}\right)$ is seen to increase in a nearly linear manner as shown in Figure 4. A similar process was conducted for a PVDF sample of $110 \mu \mathrm{m}$ thickness and $600 \AA \mathrm{NiCu}$ coat to characterize its surface morphology with external electrical potentials of 0 and $5 \mathrm{~V}$.

Figure 5. The texture change of a PVDF surface. The PVDF sample of $110 \mu \mathrm{m}$ thickness with $600 \AA \AA \mathrm{NiCu}$ coated was characterized for its surface with external electrical potentials, $0 \mathrm{~V}$ (a) and $5 \mathrm{~V}(\mathrm{~b})$.

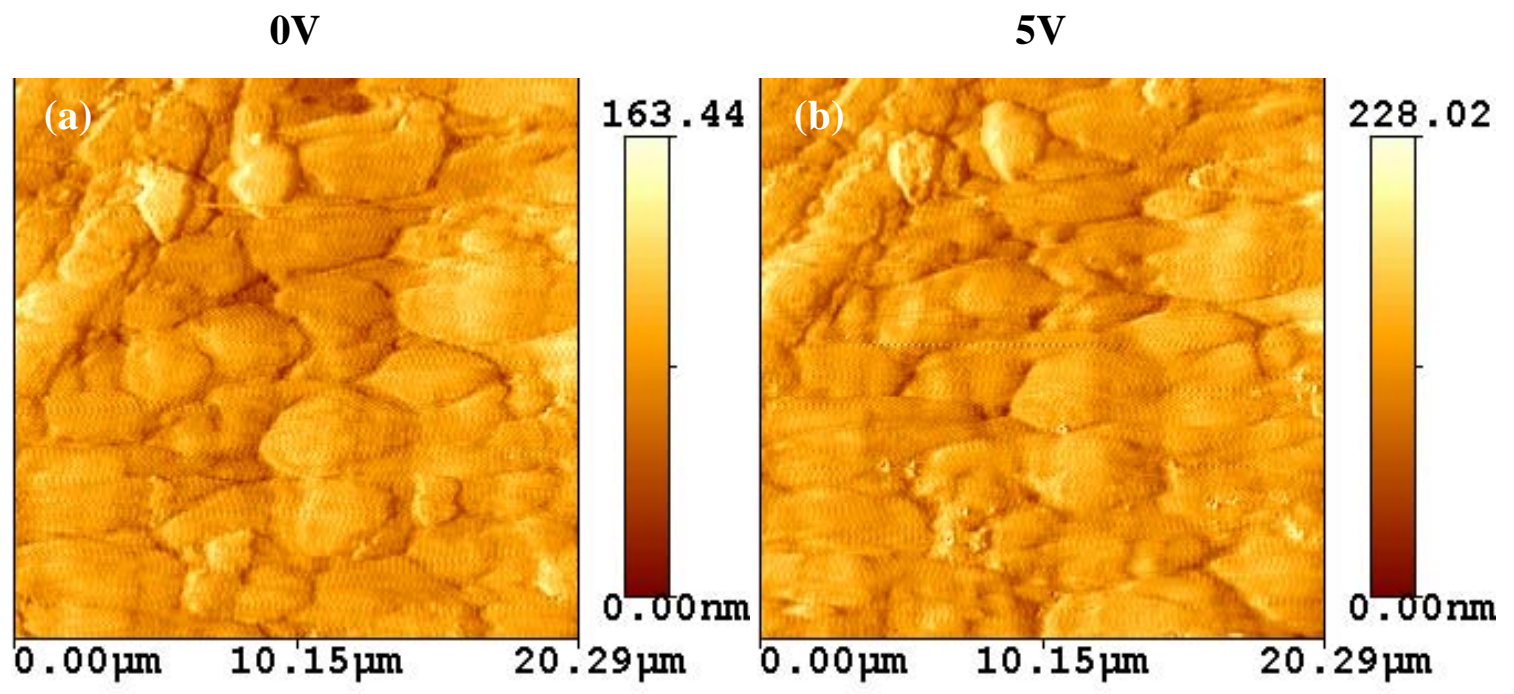

As shown in Figure 5, the surface texture was squeezed with the application of 5V. This is believed to be due to the dipole realignment resulting in an increased surface height. 
Figure 6. Investigation of the surface change rate with the applied voltage. (a) AFM images for a PVDF sample which was $52 \mu \mathrm{m}$ thickness with $150 \mathrm{~nm}$ Au coated. (b) The change of the peakto-peak distance $\left(\mathrm{D}_{\mathrm{pp}}\right)$ with the external electrical potentials.

(a)

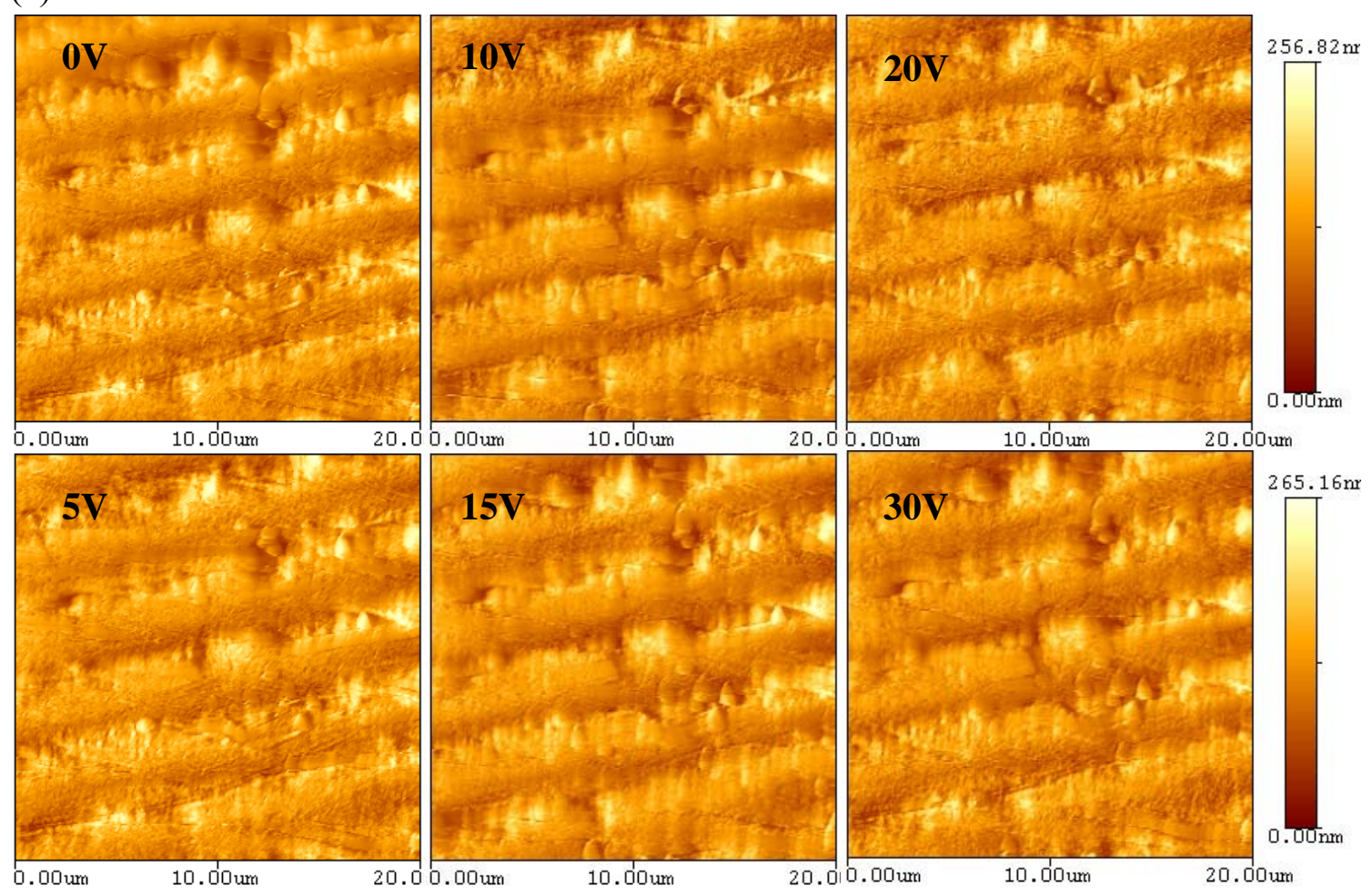

(b)

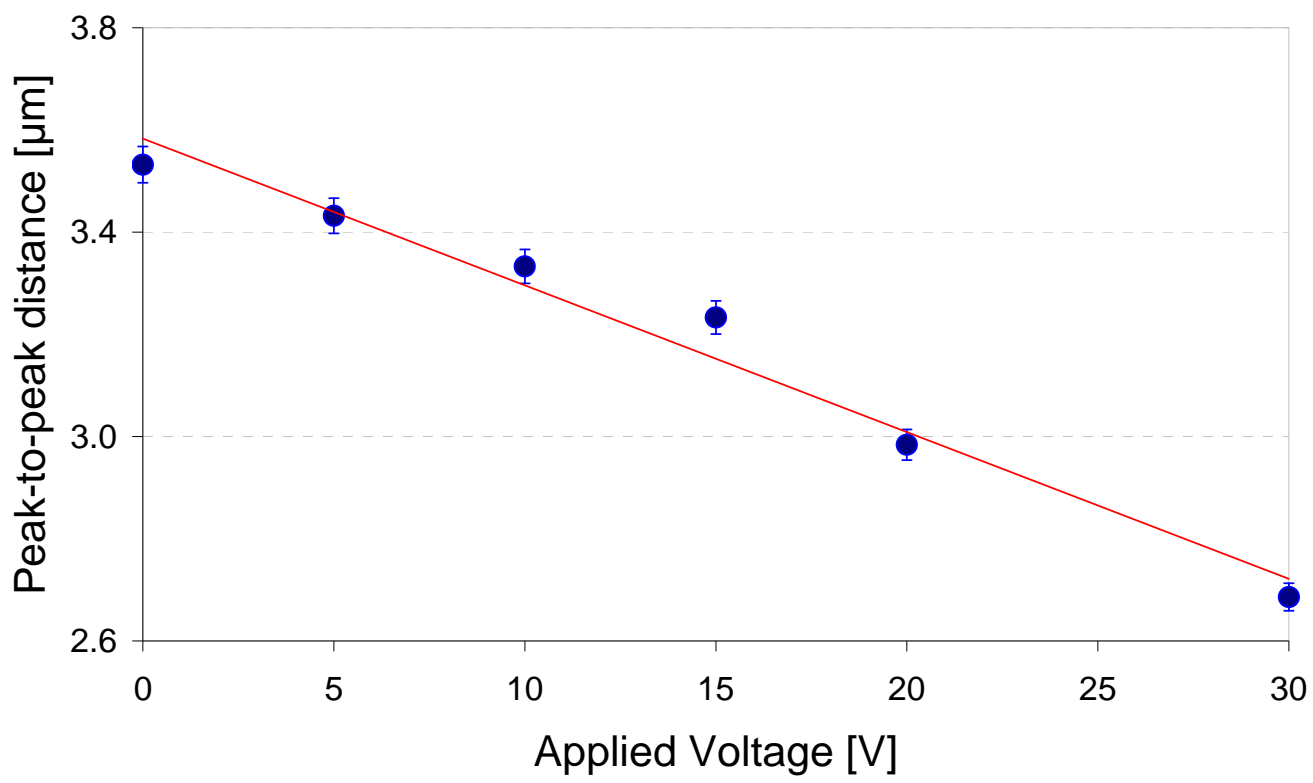

A PVDF sample with the $52 \mu \mathrm{m}$ thickness and a $150 \mathrm{~nm}$-Au-coating, was used to determine the amount of deformation caused by the applied voltage. This sample showed an original wavy surface when no voltage was applied. As a voltage was increased, the amount of squeezing deformation was observed and calculated through measuring the peak-to-peak distance $\left(D_{p p}\right)$ of the wave surface. Figure 6 (a) shows AFM images of the sample under different voltages. Figure 6(b) displays the 
change of the $\mathrm{D}_{\mathrm{pp}}$ due to external electrical potentials. As the applied electrical potential increased, the $D_{p p}$ was decreased with the rate of $28.7 \mathrm{~nm} / \mathrm{V}$. The slope of the gray line in the figure is equal to the effective piezoelectric coefficient. It is noted that without an electrical potential, the dipoles inside of the films were not aligned. They were randomly oriented and their polarity were cancelled each other so that the films were at $0 \mathrm{~V}$. When the $0 \mathrm{~V}$ was applied externally, the dipoles were aligned; negative pole was oriented to a positive charge, and positive pole to negative charge. With "zero" volt, the net voltage due to alignment was too small to be seen. However, the alignment was observed in the morphology change.

\section{Discussion}

Some polymers have showed transformation on its surface due to external stimuli [21-23]. The surface of an electro-sensitive polymer changed polymer chains and alignment with an applied electrical field [24]. Figures 3, 5, and 6 demonstrate the effect of inverse piezoelectricity due to the alignment of the dipoles in response to an applied electric potential. As a result, the potential caused the change of a surface morphology. Furthermore, the alignment of the dipoles was a temporary response to a voltage and returned to their original state after the voltage was removed. This effect is caused by lamellae (usually $20 \mathrm{~nm}$ thick) of crystalline embedded within amorphous regions (chainfolded model) of the samples. It was reported that one a 3D polymer was fixed at its ends, the local intra- and inter-chain associated orientational-deformational interactions could induce spontaneous ordering [25]. The lamellae were randomly oriented with no electric potentials present in the sample. As an electric potential was applied, the lamellae realigned to orient their dipole angle and moment correspondingly. The higher the applied potential, the greater the realignment, and in such, the greater the peak-to-valley distance. The rate of the $\mathrm{D}_{\mathrm{pp}}$ change was about $1 \mathrm{~nm}$ per $2 \mathrm{~V}$.

In order to understand the piezoelectric behavior at different length scales, we compared the charge output under stresses at nanometer and millimeter scales. The first measurement was conducted by applying external mechanical stress on the samples with the MLS shown in Figure 2. As the distance between the sample holders decreases, a mechanical stress is applied to the sample resulting in the alignment of the lamellae which produces a voltage due to the alignment of the dipoles. Figure 7(a) shows the response of such a piezo sensor. The voltage produced by the PVDF is proportional to the amount of stress applied to the sample which is proportional to the distance traveled by the MLS holders. Since the sample holder is moving in a reciprocating motion, there should be no movement at its maximum and minimum deflection thus not causing further alignment. The AFM was used to test the output of a piezo sensor when a down force was applied at one end of the polymer. Although the units of the MLS and AFM were different, the trend of their outputs was the same. This means that the localized dipole alignment is correlated with the global behavior. When an external force was applied, local or global, the PVDF produces a charge and subsequently a morphological change. The effects of electrical potential on microstructures and phase transformation of PVDF have been discussed earlier [26]. Since our focus of this research is on the surface morphological study of a piezo sensor, we will not discuss this aspect in detail. 
Figure 7. The results of (a) Motorized Linear Stage (MLS) and (b) AFM tests showing the output have similar behavior at different scales. The MLS test was to fix two ends of the sensor while the AFM was on a sensor that was fixed at one end only with manual bending.

(a) MLS bending test

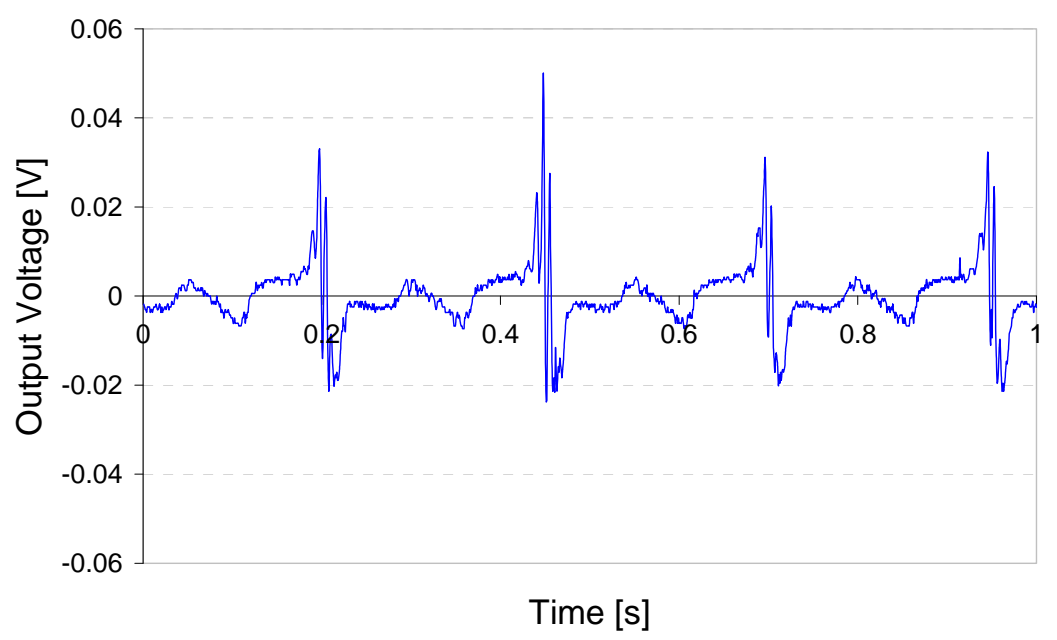

(b) AFM bending test

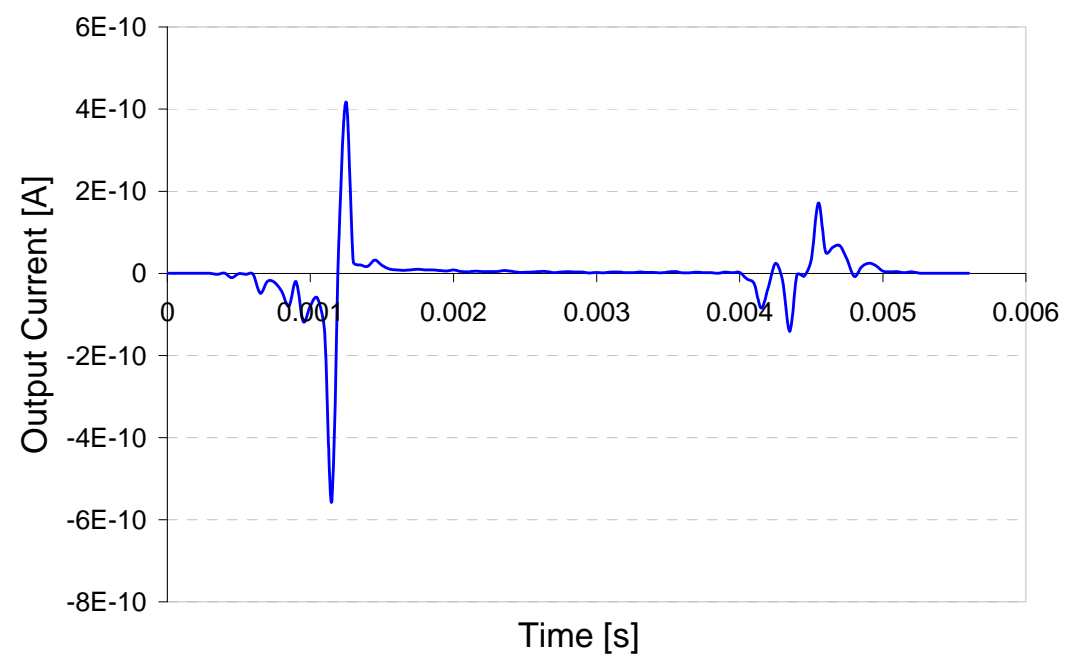

\section{Conclusions}

Metalized PVDF samples were characterized using an AFM and an external MLS in order to study the mechanisms of piezoelectric effects. Results showed that under an external potential, the surface roughness was increased. Under stress, an output was generated that is scale independent. Such variations are important for design consideration of MEMS devices and their sensitivity.

\section{Acknowledgements}

Financial support by NSF (0515930) was greatly appreciated. 


\section{References and Notes}

1 Madou, M. In Fundamentals of Microfabrication. CRC Press: Boca Raton, FL, 1997.

2 G Kovacs, G. In Micromachined Transducers Sourcebook; McGraw-Hill: New York, 1998.

3 Crane, G.R.; Comparini, A.A. Transducer applications of piezoelectric polymers. IEEE Trans. on Ind. Appl. 1977, 380-382

4 Murayama, N.; Obara, H. In Piezoelectric polymers and their applications; Tokyo, Japan, 1983; pp. 3-6.

5 Seo, I. Piezoelectric polymers and their applications. J. Jpn Soc. Precis. Eng. 1989, 55 13741347

6 Xiao, D.Q.; Lang, S.B. Measurement applications based on pyroelectric properties of ferroelectric polymers. IEEE Trans. on Electr. Insul. 1989, 24, 503-516.

7 Chen, Q.X.; Payne, P.A. Industrial applications of piezoelectric polymer transducers. Meas. Sci. Technol. 1995, 6, 249-267.

8 Aoshima, R.; Kanda, Y.; Takada, A.; Yamashita A. Sulfonated poly (vinylidene fluoride) as a biomaterial: Immobilization of urokinase and biocompatibility. J. Biomed. Mater. Res. 1982, 16, 289-299.

9 Sons, I. Polyvinylidene fluoride (PVDF) as a biomaterial: From polymeric raw material to monofilament vascular suture. J. Biomed. Mater. Res. 1995, 29, 152.51536.

10 Bouaidat, S.; Winther-Jensen, B.; Christensen, S.F.; Jonsmann, J. Plasma-polymerized coatings for bio-MEMS applications. Sens. Actuat. A, Phys. 2004, 110, 390-394.

11 Kawai, H. The piezoelectricity of poly(vinylidene fluoride). Jpn. J. Appl. Phys.1969, 8 975-976.

12 Furukawa, T.; Seo, N. Electrostriction as the origin of piezoelectricity in ferroelectric polymers Jpn. J. Appl. Phys. Part 1-Reg. Pap. Short Notes Rev. Pap. 1990, 29, 675-680.

13 Fukada, E. History and recent progress in piezoelectric polymers. IEEE Trans. Ultrason. Ferroelectr. Freq. Control 2000, 47, 1277-1290.

14 Tashiro, K.; Kobayashi, M.; Tadokoro, H.; Fukada, E. Calculation of elastic and piezoelectric constants of polymer crystals by a point-charge model - applition to poly(vinylidene fluoride) Form-1. Macromolecules 1980, 13, 691-698.

15 Tasaka, S.; Miyata, S. The origin of piezoelectricity in poly(vinylidene fluoride). Ferroelectrics 1981, 32, 17-23.

16 Tashiro, K.; Tadokoro, H.; Kobayashi, M. Structure and piezoelectricity of poly(vinylidene fluoride). Ferroelectrics 1981, 32 167-75

17 Sirohi, J.; Chopra, I. In Fundamental understanding of piezoelectric strain sensors. Technomic Publishing, University Park: PA, USA, 2000; pp. 246-257.

18 Wang, Z.Y.; Fan, H.Q.; Su, K.H.; Wen, Z.Y. Structure and piezoelectric properties of poly(vinylidene fluoride) studied by density functional theory. Polymer 2006, 47, 7988-7996.

19 Lu, F.J.; Hsu, S.L. Spectroscopic study of the electric field induced microstructural changes in poly (vinylidene fluoride). Polymer 1984, 25 1247-1252.

20 El Mohajir, B.E.; Heymans, N. Changes in structural and mechanical behaviour of PVDF with processing and thermomechanical treatments. 1. Change in structure. Polymer 2001, 42, 56615667. 
21 Sun, T.; Wang, G.; Feng, L.; Liu, B.; Ma, Y.; Jiang, L.; Zhu, D. Reversible switching between superhydrophilicity and superhydrophobicity. Angew. Chem. Int. Ed. 2004, 43, 357-360.

22 Cir, J.; Tomc, P.; Barancok, D.; Bolognesi, A.; Ragazzi, M. Dipole moment of a modified poly(3alkylthiophene) at the air/water interface. Thin Solid Films 2002, 402, 190-194

23 Duan, C.; Mei, W.N.; Yin, W.G.; Liu, J.; Hardy, J.R.; Ducharme, S.; Dowben, P.A. Simulations of ferroelectric polymer film polarization: The role of dipole interactions. Phys. Rev. B 2004, 69, 235106.

24 Song, W.; Sun, T.; Song, Y.; Bai, Y.; Liu, F.; Jiang, L. An atomic force microscopic investigation of electro-sensitive polymer surface. Talanta 2005, 67, 543-547.

25 Maksimov, A. Theory of ordering in three-dimensional polymer systems with local orientationaldeformational interactions. Polymer Sci.Ser. A 2007, 49, 599-610.

26 Wang, K.; Lee, H.; Cooper, R.; Liang, H. Time Resolved, Stress Induced, and Anisotropic Phase Transformation of a Piezoelectric Polymer. Appl. Phys. A., in press.

(C) 2008 by the authors; licensee Molecular Diversity Preservation International, Basel, Switzerland. This article is an open-access article distributed under the terms and conditions of the Creative Commons Attribution license (http://creativecommons.org/licenses/by/3.0/). 